\title{
Gender variations in the optical properties of skin in murine animal models
}

\author{
Katherine Calabro, ${ }^{a}$ Allison Curtis, ${ }^{b}$ Jean-Rene Galarneau, ${ }^{b}$ Thomas Krucker, ${ }^{b}$ and Irving J. Bigio ${ }^{a, c}$ \\ ${ }^{a}$ Boston University, Department of Biomedical Engineering, Boston, Massachusetts 02215 \\ ${ }^{b}$ Novartis Institutes for BioMedical Research, Inc., 250 Massachusetts Avenue, Cambridge, Massachusetts 02139 \\ 'Boston University, Department of Electrical and Computer Engineering, 44 Cummington Street, Boston, \\ Massachusetts 02215
}

\begin{abstract}
Gender is identified as a significant source of variation in optical reflectance measurements on mouse skin, with variation in the thickness of the dermal layer being the key explanatory variable. For three different mouse strains, the thickness values of the epidermis, dermis, and hypodermis layers, as measured by histology, are correlated to optical reflectance measurements collected with elastic scattering spectroscopy (ESS). In all three strains, males are found to have up to a $50 \%$ increase in dermal thickness, resulting in increases of up to $80 \%$ in reflectance values and higher observed scattering coefficients, as compared to females. Collagen in the dermis is identified as the primary source of these differences due to its strong scattering nature; increased dermal thickness leads to a greater photon path length through the collagen, as compared to other layers, resulting in a larger scattering signal. A related increase in the observed absorption coefficient in females is also observed. These results emphasize the importance of considering gender during experimental design in studies that involve photon interaction with mouse skin. The results also elucidate the significant impact that relatively small thickness changes can have on observed optical measurements in layered tissue. @ 2011 Society of Photo-Optical Instrumentation Engineers (SPIE). [DOI: 10.1117/1.3525565]
\end{abstract}

Keywords: mouse skin; elastic scattering spectroscopy; gender differences; layers; reflectance.

Paper 10296SSR received May 31, 2010; revised manuscript received Aug. 4, 2010; accepted for publication Aug. 12, 2010; published online Jan. 27, 2011.

\section{Introduction}

By some estimates, mice and rats account for up to $95 \%$ of all laboratory animals used in biomedical testing and research. ${ }^{1}$ This includes research in the biomedical optics field, where rodents, particularly mice, are used in the development of diagnostic and therapeutic technologies. Most of these technologies aim to be noninvasive, and thus measurements are often taken directly of or through the skin. Consequently, research that either directly or indirectly involves optical measurement of mouse skin is considerable. This includes work in photodynamic therapy, ${ }^{2,3}$ fluorescence spectroscopy, ${ }^{4}$ in-vivo bioluminescence and fluorescence imaging, ${ }^{5}$ in-vivo monitoring of hemodynamics and tumor growth, ${ }^{6,7}$ skin disease research, ${ }^{8}$ in-vivo glucose monitoring,,${ }^{9,10}$ and pharmacokinetics. ${ }^{11}$ As in all research studies, controlling for sources of biological variability is essential. When mice are involved, this entails maintaining consistency in strain, age, and housing conditions. For this work, we explore a parameter that is rarely considered in biomedical optics: gender.

The layered structure of mammalian skin consists of the epidermis, dermis, and hypodermis. The epidermis, the outer layer, is a nonvascularized stratified squamous keratinizing epithelium. In nonacral areas, especially in mice, this layer is thin (less than $20 \mu \mathrm{m})$. While keratin is a strong scatterer, its optical effect is minimal in our measurements, since the photon path length through the epidermis is so short. The dermal layer lies directly below the epidermis, and is composed of high levels of structural

Address all correspondence to: Katherine Calabro, Dept. of Biomedical Engineering, Boston University, 44 Cummington St., Boston, MA 02215. Tel: 617-3581519; E-mail: kcalabro@bu.edu. proteins, such as collagen and elastin, providing mechanical integrity and elasticity to the skin, and hair follicles in haired areas. Often referred to as the subcutaneous fat layer, the hypodermis lies beneath the dermis, and is composed primarily of adipose tissue, loose fibrous connective tissue, and larger blood vessels. ${ }^{12}$ A skeletal muscle layer, the panniculus carnosus, is usually present under the hypodermis of the mouse dorsal skin. Optically, absorption in the dermal and hypodermal layers is due to hemoglobin (both oxygenated and deoxygenated) from blood in the capillary network. Melanin also contributes to skin absorption, primarily in the epidermis and hair follicles. In the dermis, scattering is due to collagen, one of the strongest scattering structures found in soft tissue. In the hypodermis, scattering is due primarily to adipocytes. ${ }^{13,14}$

In the field of dermatology, it has long been known that male and female human skin differs significantly, primarily in the skin's overall thickness and the thicknesses of its constituent layers. ${ }^{15-21}$ Differences in the thickness of the skin layers are regulated primarily by sex hormones. ${ }^{18,19,22}$ The hypodermis is thicker in females than in males due to a greater number of fat cells. ${ }^{20}$ However, the overall thickness of male skin is greater than in females due to a higher concentration of collagen in the dermis, which has been shown to correlate with dermal thickness. ${ }^{15,21}$ Similar thickness differences have also been reported in mice, ${ }^{22-24}$ although to a much lesser extent. These differences are likely to result in variations in the observed optical characteristics of the skin. Given the extensive involvement of mouse skin in biomedical optics research, we sought to

$1083-3668 / 2011 / 16(1) / 011008 / 8 / \$ 25.00$ (C) 2011 SPIE 
study this important factor, and examine the mechanisms that underlie experimental variability of optical measurements due to variations in skin thickness and optical properties.

Motivation for this work is the result of experimental observation during a previous study ${ }^{5}$ in which optical reflectance spectra were collected from mouse skin using elastic scattering spectroscopy (ESS). ESS is a specific implementation of reflectance spectroscopy, and is a noninvasive diagnostic method used in the optical and physiological characterization of tissue (and other turbid media). It has been widely used in the diagnosis of disease, including colon cancer, ${ }^{25}$ melanoma, ${ }^{26}$ brain tumors, ${ }^{27}$ Barrett's esophagus, ${ }^{28}$ and prostate cancer. ${ }^{29}$ As opposed to diffuse reflectance spectroscopy, ESS examines light interaction when the delivery and collection probes are separated by less than one mean-free-path length, restricting interrogation to the most superficial layers, which is generally the epithelial layer, where carcinomas originate. ${ }^{30}$ For this length scale, diffusion theory is not applicable. Preliminary ESS results from the previous study of murine skin revealed significant differences between males and females, in both spectral amplitude and shape, indicating a difference in the observed optical properties of the tissue. The implications of this observation are important for any research that involves photon travel through mouse skin. In direct study of the skin, gender differences may mask other, more subtle optical changes being investigated; and for optical measurements that are collected from subdermal areas, gender variations in absorption and scattering will directly affect the amount of signal being collected through the skin. In both cases, the result can be significant experimental error if gender differences are unaccounted for. It is important to note that the results reported in this study are specific to the ESS geometry and probe dimensions employed. Measurements taken with other geometries should exhibit different results, but it is expected that the same general trends would be observed.

\section{Materials and Methods}

\subsection{Animals}

All animal handling and care was conducted in accordance with the Animal Care and Use Committee at Novartis Institutes for BioMedical Research, Incorporated, in Cambridge, Massachusetts. A total of 60 mice ( 8 weeks old) were used in this study, with equal numbers of C57B1/6 mice, CD-1 mice (Charles River Laboratories, Wilmington Massachusetts), and athymic nude mice (Harlan, Boston, Massachusetts). For each strain, ten males and ten females were used. All mice were housed under a normal 12-h light/dark cycle, and fed ad libitum standard rodent chow.

Except for the nude mice, hair was removed from the mice one day prior to experiments to reduce signal attenuation. A rectangular region of hair on the dorsal side, from the hind legs to the front legs, was first removed using an electric razor (Norelco G390, Philips, Andover, Massachusetts), and the skin was then depilated using the chemical Nair (Church and Dwight Company, Incorporated, Princeton, New Jersey). Occasional skin lesions were treated by veterinary care with triple antibiotic ointment (Taro Pharmaceuticals, Hawthorne, New York). A one-day lag was allowed for any inflammation from the hair removal process to subside.

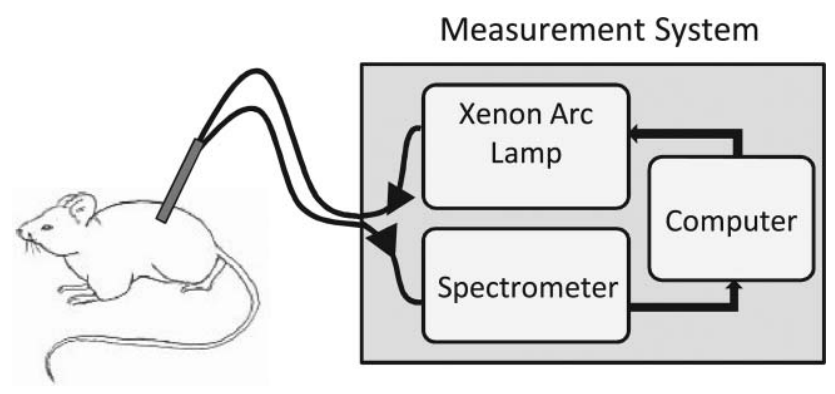

Fig. 1 Schematic of the ESS system.

\subsection{Experimental Setup and Protocol}

Figure 1 is a schematic of the elastic scattering spectroscopy system used in this study. It consists of a pulsed xenon arc lamp (Perkin Elmer LS1130-3, Waltham, Massachusetts) for the light source, a spectrometer with a linear charge-coupled device (CCD) detector (Ocean Optics S2000, Dunedin, Florida), an ADC/timer microcontroller board (TERN, Incorporated, Davis, California), and a computer interface. A fiber probe is used for delivery and collection of light; the probe used in these experiments consisted of two optical fibers with core diameters of $200 \mu \mathrm{m}$, numerical aperture of 0.22 , and a center-to-center separation of approximately $250 \mu \mathrm{m}$. A single probe was used for all measurements to avoid variability. ESS spectra were collected between 400 and $750 \mathrm{~nm}$.

All measurements were taken while the mice were under anesthesia ( $2 \%$ isoflurane in oxygen). Animals were first placed in an anesthesia induction chamber with $2 \%$ isoflurane in oxygen flowing at $150 \mathrm{ml} / \mathrm{min}$ for five minutes, and then transferred to a nose cone apparatus to maintain anesthesia levels. In collecting ESS measurements, the mice were consistently probed at three separate locations on their dorsal side: over the left and right shoulder blades, and just above the right hip. To improve optical contact between the fiber probe tip and tissue, water was applied to wet the skin surface. The locations of ESS measurements were circled for coregistration with histological examination.

After collecting ESS data, the mice were euthanized by cervical dislocation while under deep anesthesia. The dorsal skin (from the hind to front legs) was sampled, pinned to a corkboard, and fixed in $10 \%$ neutral buffered formalin. Skin samples from each of the probed areas were routinely processed to paraffin block, sectioned at $4 \mu \mathrm{m}$, and stained with hematoxylin and eosin (HE) for light microscopy examination. Each skin section was examined under light microscopy to assess the hair follicle stage. In addition, each section was scanned using a Scanscope console from Aperio (version 9.0.0.1516, Vista, California). Using the Aperio software, the thickness of the epidermis (E), epidermis plus dermis $(E+D)$, and epidermis plus dermis plus hypodermis $(\mathrm{E}+\mathrm{D}+\mathrm{H})$ were recorded.

\subsection{Data Analysis}

To extract optical and physiological information about the tissue, the collected spectra were analyzed using a previously developed empirical reflectance model. ${ }^{31}$ Briefly, the relative reflectance $R_{T}^{\mathrm{REL}}(\lambda)$ is defined as the ratio of the tissue spectrum $I_{T}(\lambda)$ to the reference spectra $I_{R}(\lambda)$ taken from a spectrally 
flat diffuse reflector (Spectralon ${ }^{\mathrm{TM}}$, Labsphere, North Sutton, New Hampshire). To eliminate variations in the relative reflectance amplitude due to variations in the distance between the probe and the Spectralon reference (measurement taken noncontact), the spectrum is normalized using the signal at $\lambda_{0}=610$ $\mathrm{nm}$ taken on a solid calibration phantom with known optical properties $I_{C}\left(\lambda_{0}\right)$ (made with titanium dioxide such that $\mu_{s}^{\prime}\left(\lambda_{0}\right)$ $\sim 10 \mathrm{~cm}^{-1}$ ). For this calibration phantom, the measurement was taken with the probe tip in contact with the phantom, using water for improved index matching. The empirical equation relating optical property values to relative reflectance values is:

$$
\begin{aligned}
R_{T}^{\mathrm{REL}}(\lambda) & =\frac{I_{T}(\lambda)}{I_{R}(\lambda)} \frac{I_{R}\left(\lambda_{0}\right)}{I_{C}\left(\lambda_{0}\right)} \\
& =a \mu_{s}^{\prime}(\lambda) \exp \left\{-C_{\text {corr }}(\lambda) \mu_{a}(\lambda) \frac{b}{\left[C_{\text {corr }}(\lambda) \mu_{a}(\lambda) \mu_{s}^{\prime}(\lambda)\right]^{c}}\right\},
\end{aligned}
$$

where $\mu_{s}^{\prime}$ and $\mu_{a}$ denote the reduced scattering and absorption coefficients, respectively. The values of $a, b$, and $c$ were determined to be $0.11,0.22$, and 0.2 , respectively, for this specific probe geometry, as described in Reif, A'Amar, and Bigio. ${ }^{31}$ The correction factor, defined by $C_{\mathrm{Corr}}(\lambda)$ is applied to account for the inhomogeneous distribution of hemoglobin in tissue, being concentrated in the blood vessels. This factor, sometimes referred to as the "compaction factor," has been derived by other groups, based on vessels modeled as infinitely long cylinders ${ }^{32}$ :

$$
C_{C o r r}(\lambda)=\left\{\frac{1-\exp \left[-2 \mu_{a, b l}(\lambda) \cdot r\right]}{2 \mu_{a, b l}(\lambda) \cdot r}\right\},
$$

where the absorption coefficient of whole blood is given by $\mu_{a, b l}$, and the mean value of the blood vessel radius is given by $r$. The optical coefficients $\mu_{s}^{\prime}(\lambda)$ and $\mu_{a}(\lambda)$ are modeled as:

$$
\begin{gathered}
\mu_{s}^{\prime}(\lambda)=d\left(\lambda^{-e}\right) \\
\mu_{a}(\lambda)=f_{1}\left[f_{2} \varepsilon_{\mathrm{HbO}}(\lambda)+\left(1-f_{2}\right) \varepsilon_{\mathrm{Hb}}(\lambda)\right],
\end{gathered}
$$

where the reduced scattering coefficient $\mu_{s}^{\prime}(\lambda)$ is modeled by a power law. Since the value $d$ has no physical meaning, its units depend directly on $e$, such that the units of $\mu_{s}^{\prime}(\lambda)$ are in inverse length (i.e., inverse centimeters). $\varepsilon_{\mathrm{HbO}}$ and $\varepsilon_{\mathrm{Hb}}$ are the extinction coefficients of oxy- and deoxyhemoglobin, respectively. The value of $e$ is a constant that depends on the mean size of the scattering particles. It can have a value ranging between 0.37 for particles much larger than the wavelength of light, and 4 for Rayleigh scattering when the particles are much smaller than the wavelength of light. ${ }^{33}$ The values of $f_{1}$ and $f_{2}$ represent the blood volume fraction and blood oxygen saturation in the tissue, respectively. The concentration of melanin was not considered in the model for several reasons. First, the levels of melanin in gray mice are expected to be negligible, since they were depilated to remove melanin from hair follicles. Further, based on visual observation, the appearance of the skin in gray mice after depilation was found to be similar to the appearance of skin in CD-1 white mice and nude mice, which is absent of melanin. Unnecessary inclusion of melanin absorption in the model would increase the number of fitting variables, limiting the accuracy of the fitting results.

The collected ESS spectra were fit to Eq. (1) using a leastsquares fit with a Levenberg-Marquardt algorithm, using $d, e$, $f_{1}, f_{2}$, and $r$ as fitting parameters that were constrained to have physically meaningful values. Matlab (Math Works, Natick, Massachusetts) was used for implementation.

\subsection{Monte Carlo}

To model the observed results, multilayer Monte Carlo simulations were performed using a program based on previous codes, ${ }^{34,35}$ and employed a variance reduction technique. ${ }^{36}$ The delivery and collection fibers were modeled to match the actual geometry of the probe used in the experiments. The fibers are both perpendicular to the surface of the material, and parallel to one another. Photon delivery and collection is restricted to the area of the fiber tip, and is constrained by the NA and index mismatch of the setup. Photon propagation in the medium is dictated by defined values for $\mu_{s}^{\prime}$ and $\mu_{a}$. The Henyey-Greenstein (HG) phase function is assumed. ${ }^{37}$ The index of refraction of the fibers and the medium were set to 1.45 and 1.35 , respectively; ${ }^{38}$ however, variation in the refractive index of the medium between 1.34 and 1.38 caused only minor differences in results. Simulations were terminated once 10,000 photons were successfully collected by the collection fiber.

\section{Results}

\subsection{Histological Skin Thickness Measurements}

Figure 2 plots the averages of thickness values for each layer of mouse skin, as determined by histology. Data are separated by strain and gender (Gray refers to C57Bl/6 mice, and White refers to CD-1 mice), and are consistent with previous findings in mice. ${ }^{22}$ For all strains, the difference in the overall thickness of the skin between males and females is statistically significant. (Throughout this work, statistical significance was determined using the unpaired t-test, with $p$ values less than 0.01 being considered significant.) The thickness of the dermis is the most substantial difference between genders for all three strains. Gender difference in the thickness of the hypodermis is statistically significant only for gray mice, while gender

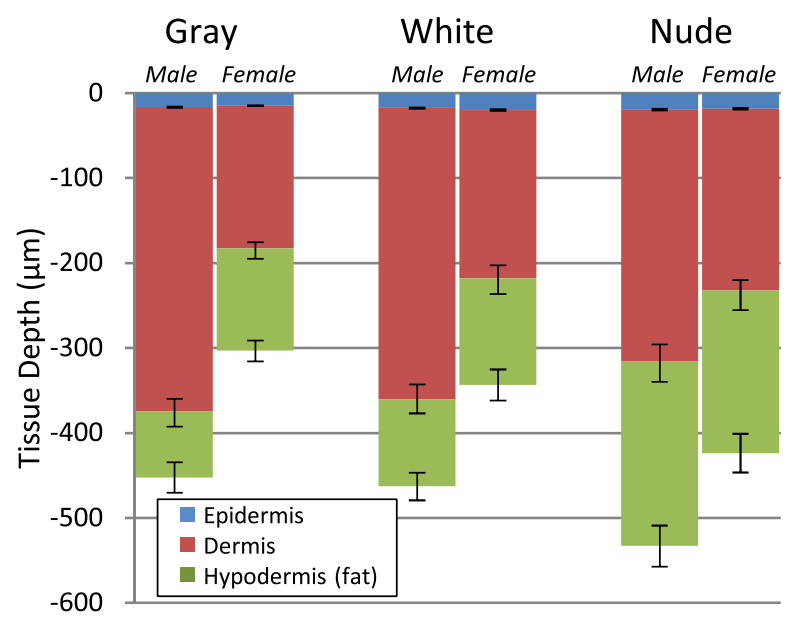

Fig. 2 Averaged values of the epidermis, dermis, and hypodermis thicknesses as measured by histology, separated by strain and gender. Gray refers to $\mathrm{C} 57 \mathrm{Bl} / 6$ mice, and White refers to CD-1 mice. Variation in the thickness of the dermis is the most significant difference between genders. Error bars correspond to the standard error of the mean. 
Table 1 The distribution of animals in each hair growth phase, separated by strain and gender, demonstrates that there is no gender bias based on hair growth cycle.

\begin{tabular}{|c|c|c|c|c|c|c|}
\hline & \multicolumn{2}{|c|}{ Gray } & \multicolumn{2}{|c|}{ White } & \multicolumn{2}{|c|}{ Nude } \\
\hline & Male & Female & Male & Female & Male & Female \\
\hline Telogen & 4 & 4 & 6 & 5 & 0 & 0 \\
\hline Catagen & 6 & 6 & 4 & 5 & 1 & 2 \\
\hline Anagen & 0 & 0 & 0 & 0 & 9 & 8 \\
\hline
\end{tabular}

differences in epidermal thickness were not significant for any strain. As a general trend, the $p$ values comparing gender differences are most significant for gray mice $(p<1 \mathrm{e}-8)$, and are least significant for nude mice $(0.001<p<0.002)$, indicating that gender differences are most prominent in gray mice. Because hair follicle stage also influences skin thickness, ${ }^{39}$ we ensured that the observed thickness differences were not gender biased based on hair cycle. Microscopic assessment of skin samples demonstrated a comparable distribution of anagen, telogen, and catagen hair cycle stages between strain-matched male and female mice (see Table 1).

\subsection{Elastic Scattering Spectroscopy Reflectance Measurements and Extracted Optical Properties}

The average ESS spectra for males and females of each strain are presented in Fig. 3. The red and blue lines represent female and male mice, respectively (color online only). The solid, dashed, and dotted curves represent gray, white, and nude mice, respectively. Note that the difference between male and female spectra is greatest for gray mice, and smallest for nude mice. This is consistent with the degree of difference between genders in the layer thickness values.

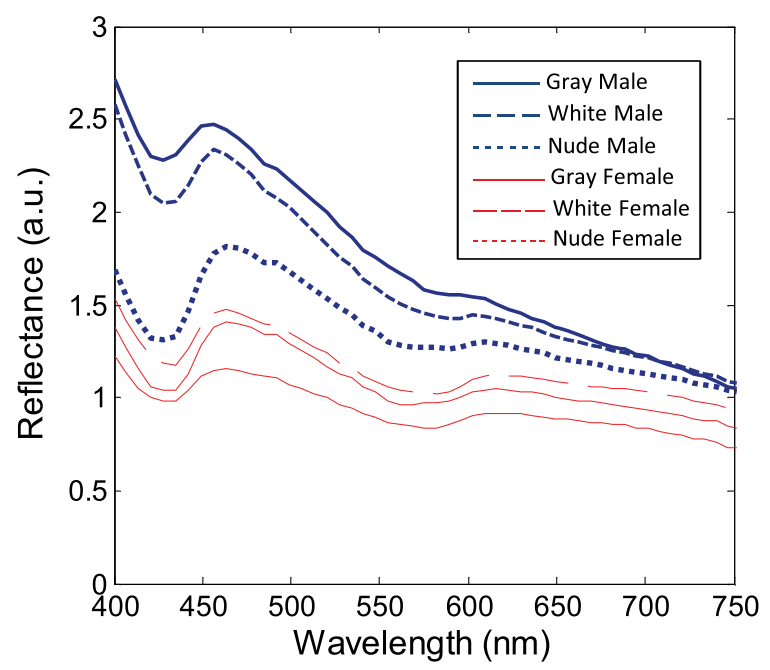

Fig. 3 Relative reflectance spectra of skin, averaged for each gender and strain. Differences in amplitude and shape indicate significant difference in the optical properties.
To understand the physiological differences that lead to these spectral variations, the previously described empirical model was used to calculate (from the ESS spectra) the average optical properties of the skin. Figure 4 presents the average extracted values of the reduced scattering and absorption coefficients $\mu_{s}^{\prime}$ and $\mu_{a}$, along with the measured relative reflectance values $\left[R_{T}^{\mathrm{REL}}(\lambda)\right]$. These values are reported at the wavelength $\lambda=550 \mathrm{~nm}$, which falls within the hemoglobin absorption q-bands. Note that because the reduced scattering coefficient $\mu_{s}^{\prime}$ is linearly proportional to relative reflectance, ${ }^{31}$ the scattering coefficient and reflectance values follow similar trends.

The gender differences in reflectance and scattering values are significant for all three strains, with gray mice showing the greatest significance $(p<2 \mathrm{e}-10)$. We posit that this difference is due to the larger dermal thickness in males as compared to females; an increase in dermal thickness increases the photon path length in the dermis, and decreases the path length in the hypodermis and structural muscle layers (assuming similar total path lengths and penetration depths). The total collected reflectance signal is thus composed of a larger contribution from the dermis, and the observed average scattering coefficient is weighted more heavily by the optical properties of that layer. Given the strong scattering properties of collagen in the dermis, this leads to higher reflectance and scattering coefficient values. In terms of the fitting parameters in Eq. (3), the increase in reduced scattering coefficient is due to an average $81 \%$ increase in the value of $c$, and an average $24 \%$ increase in the value of $d$ in males as compared to females.

For the absorption coefficient values, strong statistical significance was observed only in gray mice $(p=1 \mathrm{e}-7)$, while the gender differences for white and nude mice were marginal ( $p=0.019$ and $p=0.0043$, respectively). The increase in absorption observed in females is due to an average $28 \%$ increase in blood volume fraction $\left(f_{1}\right)$ compared to males, as determined from the spectral analysis. We deduce that this is from increased blood profusion in either the hypodermis fat layer, the subjacent structural muscle layer, or both, as compared to the dermis. With an increased proportion of the path length lying in the hypodermis and muscle layers, increased hemoglobin/myoglobin absorption in these deeper layers results in slightly higher absorption coefficient values as compared to males. However, we emphasize that this effect is small; the strongest source of signal variation is due to differences in scattering.

To better visualize the relationship of dermal thickness and observed optical properties, scatter plots are presented in Fig. 5. Because gray mice were observed to have the greatest gender differences in both thickness values and optical properties, we use their data to illustrate these relationships. In both plots of Fig. 5, the data points of males and females are well separable. The positive correlation between dermal thickness and scattering, and the negative correlation between dermal thickness and absorption in Figs. 5(a) and 5(b), respectively, support the previous conclusions that measured scattering and absorption values are a function of the thickness of the dermis. The relationships between hypodermis thickness and optical properties (not shown) were not found to have a strong correlation, indicating that the thickness of the hypodermis does not significantly impact reflectance signal variations. Additionally, relationships between the total skin thickness (epidermis + dermis + hypodermis) and optical properties (not shown) reveal very 

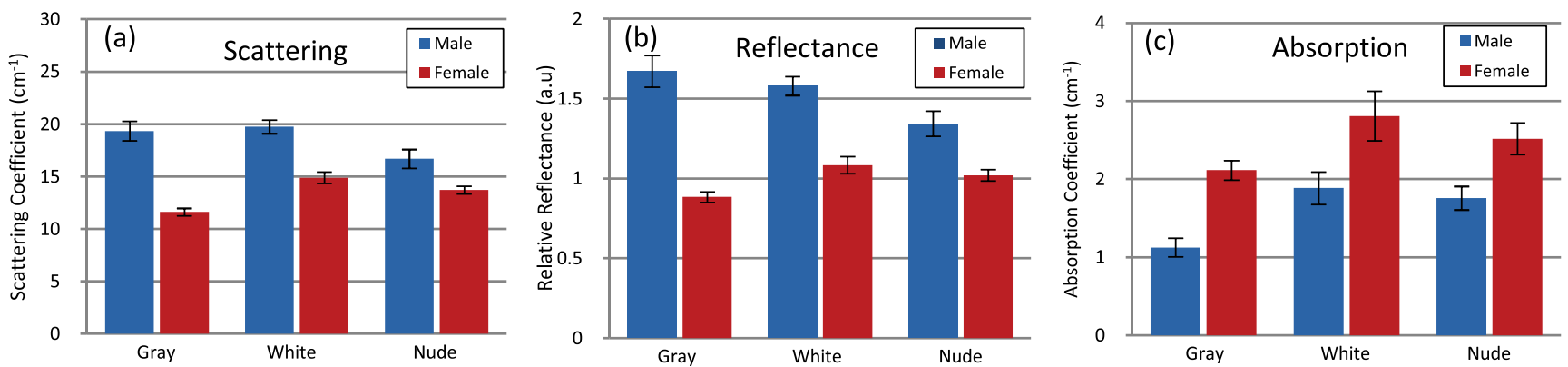

Fig. 4 Averaged values of the measured reflectance, and calculated scattering and absorption coefficients, separated by strain and gender at the wavelength $550 \mathrm{~nm}$. Scattering and reflectance values are the most significant gender differences; gender difference in absorption is moderate. Error bars correspond to the standard error of the mean.

similar trends to those in Fig. 5, confirming that the dominant variable is the thickness of the dermis as opposed to the hypodermis. It also suggests that variation in photon path length in the muscle layer does not greatly contribute to reflectance variation for the fiber probe geometry used here.
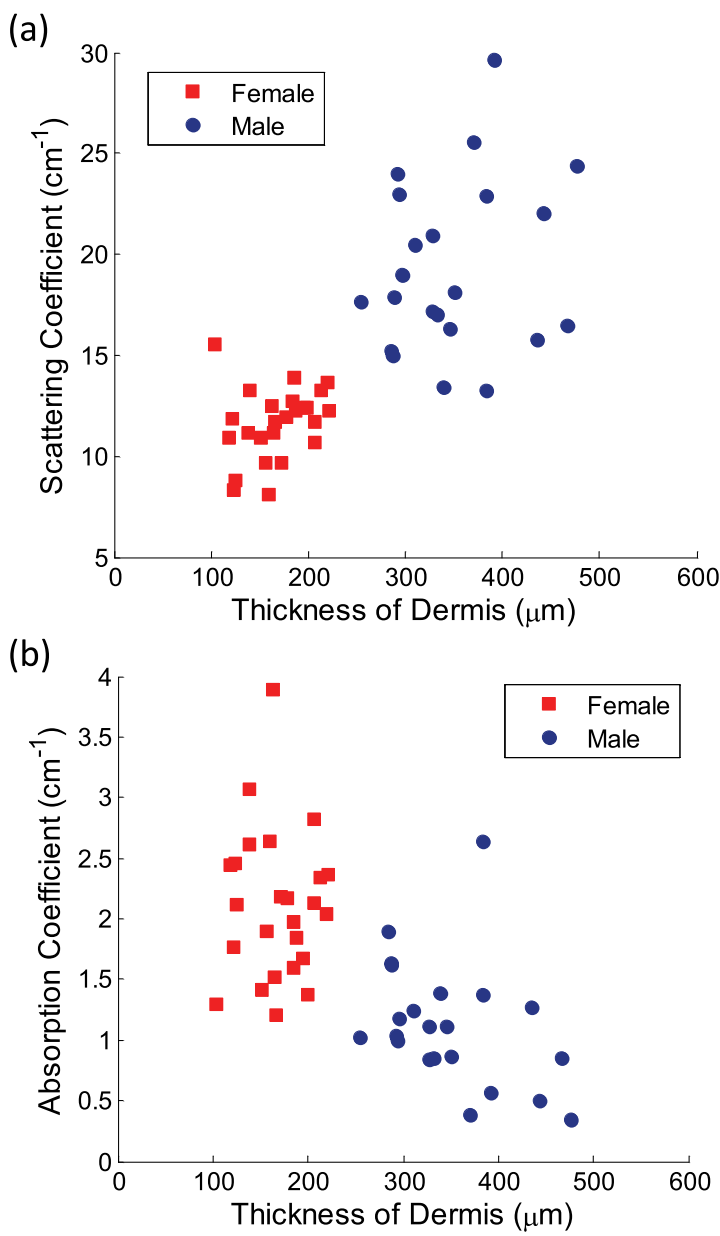

Fig. 5 Scatter plots relating dermal thickness to observed scattering and absorption coefficients for gray mice at the wavelength $550 \mathrm{~nm}$. Male and female data points are well separable and illustrate (a) positive correlation between dermis thickness and reduced scattering coefficient and (b) negative correlation between dermis thickness and absorption coefficient.

\subsection{Monte Carlo Simulations}

Monte Carlo simulations were run to further validate our conclusions concerning the mechanisms underlying gender variations in ESS reflectance signals. The tissue structure was modeled with three layers. The top layer represents the combined epidermis and dermis. Because of its small thickness, and thus minimal optical contribution, the epidermis is grouped with the dermis. Further, the optical properties in the epidermis have been reported to be similar to those in the dermis. ${ }^{40}$ The middle layer represents the hypodermis fat layer, and the bottom layer represents the muscle below the skin. Thickness values for the dermis and hypodermis layers were chosen to cover the range of values observed in histology (Fig. 2), while the muscle layer was modeled to be semi-infinite. For dermal thickness, five values were chosen: 200, 250, 300, 350, and $400 \mu \mathrm{m}$. For each dermis thickness value, four values of hypodermis thickness were chosen: $100,150,200$, and $250 \mu \mathrm{m}$. This resulted in a set of 20 simulations. Six additional simulations were also run, corresponding to the average dermis and hypodermis thickness values observed in males and females of each strain (Fig. 2). Unfortunately, representative optical property values could not be obtained from the literature because there are few sources that report on the optical properties of mouse skin, ${ }^{41,42}$ and none that report on the separate property values for the individual layers of skin. While the optical properties of skin layers in humans cannot be directly assumed for mice, these values were used as guidelines in defining the relative differences among layers. ${ }^{13,40,43-45}$ Hence, optical property values at the wavelength $550 \mathrm{~nm}$ were defined for each of the layers as: $\mu_{a}=2,3$, and $3 \mathrm{~cm}^{-1}$, and $\mu_{s}^{\prime}=27,14$, and $7.5 \mathrm{~cm}^{-1}$, for the dermis, hypodermis, and muscle layers, respectively. Note that for our purpose of observing reflectance trends, the actual values of the properties are less important than the relative differences between them.

Figure 6 illustrates the results of the simulations in a plot relating the total thickness of the skin (dermis + hypodermis) to reflectance values. The data are separated into five sets, each representing a different dermal thickness. For each of these sets, the data points from left to right on each curve represent different hypodermis thickness values, from smallest $(100 \mu \mathrm{m})$ to largest $(250 \mu \mathrm{m})$. The reflectance values from the simulations representing the males and females in this study are also plotted, illustrating a similar gender separation as observed in Fig. 5(a). Confirming experimental conclusions, the primary variable in 


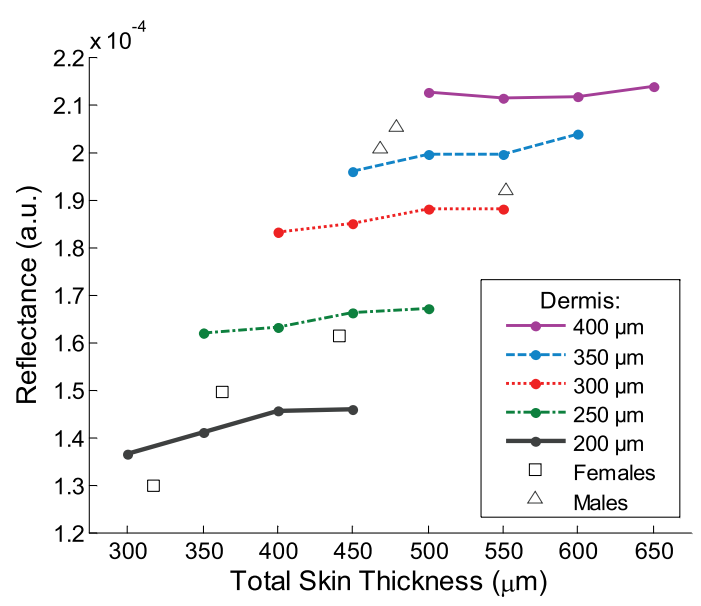

Fig. 6 Monte Carlo simulation data: reflectance versus total skin thickness (dermis + hypodermis). Data are separated into five sets, each representing a different dermal thickness. For each set, the data points from left to right on each curve represent different hypodermis thicknesses, from 100 to $250 \mu \mathrm{m}$. Representative reduced scattering and absorption coefficients at $550 \mathrm{~nm}$ for each skin layer were used in the simulations. Reflectance values from the simulations representing the male and female geometries measured experimentally are plotted as triangles and squares, respectively. Results show that dermal thickness impacts reflectance much more than hypodermal thickness.

the determination of reflectance is shown to be the thickness of the dermis. Conversely, the thickness of the hypodermis plays only a minor role; for each dermis thickness, an increase in hypodermis thickness produces only a marginal increase in reflectance. This increase is the result of the hypodermis having a higher scattering coefficient value than the muscle layer. The effects of absorption on the simulations were also examined by performing the simulations with different absorption coefficient values. Changes in absorption coefficient values of up to $50 \%$ in the hypodermis and subjacent skeletal muscle layers did not result in significant changes to reflectance, supporting the minimal correlation between absorption coefficient and layer thickness observed experimentally. It should be noted that all of these parametric dependences of reflectance on skin layer properties are specific to the optical geometry described here. Results are likely to change for different fiber separation, NA, angle of incidence, etc., but the general trends are expected to hold.

Figure 7 illustrates the percentage of the path length traveling through each layer of the skin as the dermal thickness increases. This plot indicates that more than $60 \%$ of the photon path length occurs in the dermis, while path lengths through the hypodermis and muscle layers each account for less than $20 \%$. These data assume a constant hypodermal thickness of $200 \mu \mathrm{m}$; variations in hypodermis thickness result in minimal differences to the plot. Because the signal contribution from the dermal layer is so much larger, changes in its thickness are much more influential than changes in hypodermis thickness. This clarifies why dermal thickness plays such a dominant role in the variation of reflectance. In comparing the percentage path length values for the simulations representing male and female geometries in our experimental study, an average increase in dermal path length percentage from $67 \%$ in females to $88 \%$ in males results in an average reflectance increase of $40 \%$. An alternative approach

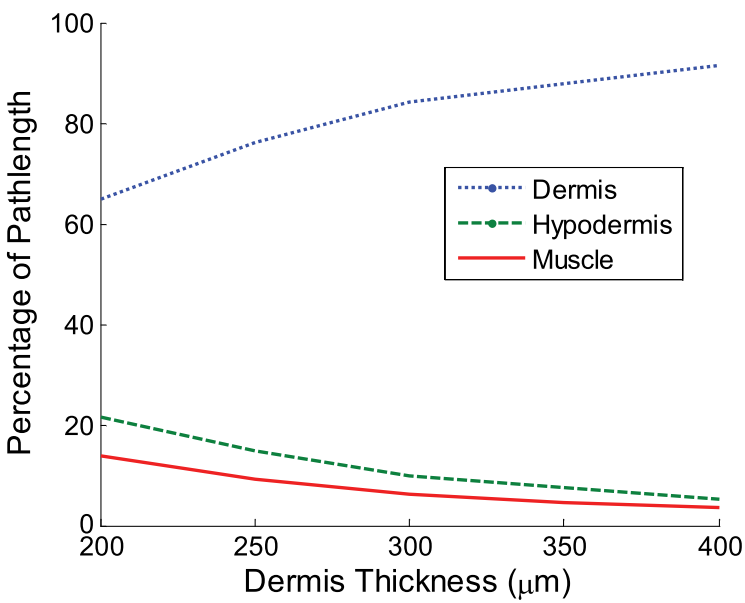

Fig. 7 The percentage of photon path length in each layer. More than $60 \%$ of the path length resides in the dermis, and increases to more than $90 \%$ as its thickness increases. This large contribution causes small changes in the dermis geometry to result in more drastic reflectance differences than would occur for layers with less percentage path lengths. Representative reduced scattering and absorption coefficients at $550 \mathrm{~nm}$ for each skin layer were used in the simulations along with a hypodermal thickness of $200 \mu \mathrm{m}$.

to conceptualizing these results is to consider the hypodermis and muscle layers as a "photon-sink." Once a photon reaches the hypodermis, it experiences much less scattering because of the lower scattering coefficient values of the subdermal layers. Consequently, it has a low enough probability of scattering to reverse the direction back toward the collection probe before it is absorbed.

\section{Discussion}

In this work, experimental variation in optical measurements from the skin of mice was examined as a function of gender. However, other factors such as location on the body, age, hydration level, and hair growth phase have also been reported as factors influencing skin thickness. ${ }^{39,46-48}$ These additional factors likely contributed to the experimental variability observed in our study, but were likely less significant than layer thickness differences; as was presented in the results, the influence of hair growth phase was specifically investigated to ensure that there was no gender bias based on hair cycle. It is also important to consider that gender differences in the skin may not be limited to changes in geometrical thickness. Variations in the physical and optical properties of the skin are possible, and would also result in measurement variability, independent of layer thickness. Some factors that would alter the optical properties of the skin include variation in collagen density, ${ }^{15}$ larger sebaceous gland size in males, ${ }^{23}$ and variations in blood volume based on vasoconstriction, vasodilatation, or opening of reserve capillaries, all in response to various stimuli (e.g., heat, cold, irritation, exercise, hypotension, etc.). However, as demonstrated in the Monte Carlo simulations, variation in layer thickness is more influential than variation in optical property values. These confounding factors highlight the challenges involved in biological measurements, and underscore the importance of proper experimental controls; even unlikely factors can result in measurement 
variability, which may be significant enough to obscure desired information.

While the trends observed here are likely to be observed in other optical applications, the degree of observed optical variation will differ depending on measurement geometry. In general, variations will be considerable when a significant percentage of the path length is in the skin, and when the optical path travels through multiple layers. These conditions are often achieved when measurements are taken in the reflection geometry with diffuse illumination. Alternatively, in applications such as fluorescence spectroscopy and bioluminescence imaging, in cases where the source of collected light is deeper in the tissue, the skin only accounts for a small percentage of the total optical path length. In this case, differences in dermal thickness will have much less impact on the collected signal than other variables. Given the probe geometry used in this study, little gender variation would be expected in measurements of human skin, since the combined thickness of the epidermis and dermis is much thicker than in mice, and thus very little, if any, of the optical signal will be collected from a secondary layer. It is plausible, however, that gender variations would be significant when probes are used that have larger source detection separations, and signal penetration is deeper.

Aside from identifying the importance of gender in optical measurement variability in mouse skin, these results highlight the more general concern of optical measurement variation in any layered tissue. When identifying epithelial disease, for example, differences in spectra are considered to be the result of variation in the local optical properties due to histological changes at the cellular level. However, if layer thicknesses are not consistent among samples, large spectral changes may be the result of geometry changes, and differences in observed optical properties would be based on path length variations, and not necessarily disease. Thus, in layered tissues, it is important to distinguish microscopic changes based on disease from macroscopic changes based on structure.

\section{Conclusion}

In this work, we identify gender as a significant source of variation in optical reflectance measurements of mouse skin, and illustrate that variation in the thickness of the dermal layer is the key explanatory variable. For three strains of mice, the thickness values of the epidermis, dermis, and hypodermis layers, as measured by histology, are correlated to optical reflectance measurements collected with elastic scattering spectroscopy (ESS). Males are found to have significantly thicker dermal layers, resulting in increases of up to $80 \%$ in reflectance as compared to females. As a result, collagen in the dermis is identified as the source of these differences due to its strong scattering properties. It is concluded that increased dermal thickness results in longer photon path lengths through the collagen, which produces a larger scattering signal. A small increase in the observed absorption coefficient in females is also observed, resulting from a longer path length in the lower layers, and a presumed greater blood volume fraction in the hypodermis and/or subdermal muscle layers. Monte Carlo simulations that model thickness changes in a layered structure support our conclusions. These results emphasize the importance of considering gender during experimental design of any study that involves photon interaction with mouse skin. They also emphasize the significant impact that relatively small thickness changes can have on observed optical measurements in layered tissues.

\section{Acknowledgments}

This work was supported in part by the Boston University Photonics Center and the CIMIT consortium.

\section{References}

1. F. L. Trull and B. A. Rich, "More regulation of rodents," Science 284, 1463 (1999).

2. M. A. Middelkamp-Hup, I. Sánchez-Carpintero, S. Kossodo, P. Waterman, S. González, M. C. Mihm, Jr., and R. R. Anderson, "Photodynamic therapy for cutaneous proliferative vascular tumors in a mouse model," J. Invest. Dermatol. 121, 634-639 (2003).

3. D. Robinson, H. S. de Bruijn, N. Van Der Veen, M. R. Stringer, S. B. Brown, and W. M. Star, "Protoporphryin IX fluorescence photobleaching during ALA-mediated photodynamic therapy of UVB-induced tumors in hairless mouse skin," Photochem. Photobiol. 69, 61-70 (1999).

4. D. S. Gareau, P. R. Bargo, W. A. Horton, and S. L. Jacques, "Confocal fluorescence spectroscopy of subcutaneous cartilage expressing green fluorescent protein versus cutaneous collagen autofluorescence," J. Biomed. Opt. 9(2), 254-258 (2004).

5. A. Curtis, K. W. Calabro, J. Galarneau, I. J. Bigio, and T. Krucker, "Temporal variations of skin pigmentation in C57Bl/6 mice affect optical bioluminescence quantitation," Mol. Imaging. Biol. published online (2010).

6. M. Xia, V. Kodibagkar, H. Liu, and R. P. Mason, "Tumor oxygen dynamics measured simultaneously by near-infrared spectroscopy and $19 \mathrm{~F}$ magnetic resonance imaging in rats," Phys. Med. Biol. 51, 45-60 (2006).

7. G. M. Palmer, R. J. Viola, T. Schroeder, P. S. Yarmolenko, M. W. Dewhirst, and N. Ramanujam, "Quantitative diffuse reflectance and fluorescence spectroscopy: tool to monitor tumor physiology in vivo," J. Biomed. Opt. 14(2), 024010 (2009).

8. M. Amouroux, G Díaz-Ayil, W. C. P. M. Blondel, G. Bourg-Heckly, A. Leroux, and F. Guillemin, "Classification of ultraviolet irradiated mouse skin histological stages by bimodal spectroscopy: multiple excitation autofluorescence and diffuse reflectance," J. Biomed. Opt. 14(1), 014011 (2009).

9. M. Kinnunen, R. Myllylä, and S. Vainio, "Detecting glucose-induced changes in in vitro and in vivo experiments with optical coherence tomography," J. Biomed. Opt. 13(2), 021111 (2008).

10. Handbook of Optical Sensing of Glucose in Biological Fluids and Tissues, V. V. Tuchin Ed., CRC Press, Taylor \& Grancis Group, London (2009).

11. J. McMurdy, J. Reichner, A. Mathews, M. Markey, S. Intwala, and G. Crawford, "Broadband reflectance spectroscopy for establishing a quantitative metric of vascular leak using the Miles assay," J. Biomed. Opt. 14(5), 054012 (2009).

12. J. C. Peckham and K. Heider, "Skin and subcutis," in Pathology of the Mouse, R. R. Maronpot, Ed., pp. 555-635, Cache River Press, St. Louis, MO (1999).

13. A. N. Bashkatov, E. A. Genina, V. I. Kochubey, and V. V. Tuchin, "Optical properties of human skin, subcutaneous and mucous tissues in the wavelength range from 400 to $2000 \mathrm{~nm}$, , J. Phys. D: Appl. Phys. 38, 2543-2555 (2005).

14. S. Tseng, A. Grant, and A. J. Durkin, "In vivo determination of skin near-infrared optical properties using diffuse optical spectroscopy," J. Biomed. Opt. 13(1), 014016 (2008).

15. S. Shuster, M. M. Black, and E. McVitie, "The influence of age and sex on skin thickness, skin collagen and density," Brit. J. Dermatol. 93, 639-643 (1975).

16. H. Dao Jr. and R. A. Kazin, "Gender differences in skin: a review of the literature," Gender Med. 4, 308-328 (2007).

17. E. Tur, "Physiology of the skin-differences between women and men," Clin. Dermatol. 15, 5-16 (1997). 
18. M. Brincat, S. Kabalan, J. W. Studd, C. F. Moniz, J. de Trafford, and J. Montgomery, "A study of the decrease of skin collagen content, skin thickness and bone mass in the postmenopausal woman," Obstet. Gynecol. 70, 840-845 (1987).

19. C. Eisenbeiss, J. Welzel, and W. Schmeller, "The influence of female sex hormones on skin thickness: evaluation using $20 \mathrm{MHz}$ sonography," Brit. J. Dermatol. 139, 462-467 (1998).

20. L. Sjöström, U. Smith, M. Krotkiewski, and P. Björntorp, "Cellularity in different regions of adipose tissue in young men and women," Metabolism 21, 1143-1153 (1972).

21. S. Seidenari, A. Pagnoni, A. D. di Nardo, and A. Giannetti, "Echographic evaluation with image analysis of normal skin: variations according to age and sex," Skin Pharmacol. 7, 201-209 (1994).

22. L. Azzi, M. El-Alfy, C. Martel, and F. Labrie, "Gender differences in mouse skin morphology and specific effects of sex steroids and dehydroepiandrosterone," J. Invest. Dermatol. 124, 22-27 (2005).

23. L. Azzi, M. El-Alfy, and F. Labrie, "Gender differences and effects of sex steroids and dehydroepiandrosterone on androgen and oestrogen alpha receptors in mouse sebaceous glands," Brit. J. Dermatol. 154, 21-27 (2006).

24. S. Movérare, M. K. Lindberg, J. Faergemann, J. Å Gustafsson, and C. Ohlsson, "Estrogen receptor alpha, but not estrogen receptor beta, is involved in the regulation of the hair follicle cycling as well as the thickness of epidermis in male mice," J. Invest. Dermatol. 119, 10531058 (2002).

25. G. Zonios, L. T. Perelman, V. Backman, R. Manoharan, M. Fitzmaurice, J. Van Dam, and M. S. Feld, "Diffuse reflectance spectroscopy of human adenomatous colon polyps in vivo," Appl. Optics 38, 6628-6637 (1999).

26. G. Zonios, A. Dimou, I. Bassukas, D. Galaris, A. Tsolakidis, and E. Kaxiras, "Melanin absorption spectroscopy: new method for noninvasive skin investigation and melanoma detection," J. Biomed. Opt. 13(1), 014017 (2008).

27. M. Canpolat, M. Akyüz, G. Gürer, and R. Tuncer, "Intra-operative brain tumor detection using elastic light single-scattering spectroscopy: a feasibility study," J. Biomed. Opt. 14(5), 054021 (2009).

28. L. B. Lovat, K. Johnson, G. D. Mackenzie, B. R. Clark, M. R. Novelli, S. Davies, M. O'Donovan, C. Selvasekar, S. M. Thorpe, D. Pickard, R. Fitzgerald, T. Fearn, I. Bigio, and S. G. Brown, "Elastic scattering spectroscopy accurately detects high grade dysplasia and cancer in Barrett's oesophagus," Gut 55, 1078-1083 (2006).

29. I. J. Bigio, O. A’Amar, and M. S. Hirsch, "Elastic scattering spectroscopy for detection of prostate cancer: preliminary feasibility study," Proc. SPIE 5141, 142-146 (2003).

30. A. M. J. Wang, J. E. Bender, J. Pfefer, U. Utzinger, and R. A. Drezek, "Depth-sensitive reflectance measurements using obliquely oriented fiber probes," J. Biomed. Opt. 10(4), 044017 (2005).

31. R. Reif, O. A'Amar, and I. J. Bigio, "Analytical model of light reflectance for extraction of optical properties in small volumes of turbid media," Appl. Optics 46, 7317-7328 (2007).

32. R. L. P. van Veen, W. Verkruysse, and H. J. C. M. Sterenborg, "Diffuse-reflectance spectroscopy from 500 to $1060 \mathrm{~nm}$ by correction for inhomogeneously distributed absorbers," Opt. Lett. 27, 246-248 (2002).
33. R. Graaff, J. G. Aarnoudse, J. R. Zijp, P. M. A. Sloot, F. F. M. de Mul, J. Greve, and M. H. Koelink, "Reduced light-scattering properties for mixtures of spherical particles: a simple approximation derived from Mie calculations," Appl. Optics 31, 1370-1376 (1992).

34. S. L. Jacques, and L. Wang, "Monte Carlo modeling of light transport in tissue," in Optical-Thermal Response of Laser Irradiated Tissue, A. J. Welch and M. J. C. van Germet, Eds., pp. 73-100, Plenum, New York (1995).

35. L. H. Wang, S. L. Jacques, and L. Zheng, "MCML: Monte-Carlo modeling of light transport in multilayered tissues," Comput. Meth. Prog. Biol. 47, 131-146 (1995).

36. M. Hiraoka, M. Firbank, M. Essenpreis, M. Cope, S. R. Arridge, P. van der Zee, and D. T. Delpy, "A Monte Carlo investigation of optical path length in inhomogeneous tissue and its application to near-infrared spectroscopy," Phys. Med. Biol. 38, 1859-1876 (1993).

37. L. G. Henyey and J. L. Greenstein, "Diffuse radiation in the galaxy," Astrophys. J. 93, 70-83 (1941).

38. H. Ding, J. Q. Lu, W. A. Wooden, P. Kragel, and X. Hu, "Refractive indicies of human tissues at eight wavelengths and estimated dispersion relationships between 300 and 1600nm," Phys. Med. Biol. 51, 14791489 (2006).

39. L. S. Hansen, J. E. Coggle, J. Wells, and M. W. Charles, "The influence of the hair cycle on the thickness of mouse skin," Anat. Rec. 210, 569-573 (1984).

40. C. R. Simpson, M. Kohl, M. Essenpreis, and M. Cope, "Near-infrared optical properties of ex vivo human skin and subcutaneous tissues measured using the Monte Carlo inversion technique," Phys. Med. Biol. 43, 2465-2478 (1998).

41. R. Samatham, S. L. Jacuques, and P. Campagnola, "Optical properties of mutant versus wild-type mouse skin measured by reflectance-mode confocal scanning laser microscopy (rCSLM)," J. Biomed. Opt. 13(4), 041309 (2008).

42. W. Cheong, S. A. Prahl, and A. J. Welch, "A review of the optical properties of biological tissues," IEEE J. Quant. Elect. 26, 2166-2185 (1990).

43. I. V. Meglinski and S. J. Matcher, "Quantitative assessment of skin layers absorption and skin reflectance spectra simulation in the visible and near-infrared spectral regions," Physiol. Meas. 23, 741-753 (2002).

44. S. Tseng, P. Bargo, A. Durkin, and N. Kollias, "Chromophore concentrations, absorption, and scattering properties of human skin in-vivo," Opt. Express 17, 14599-14617 (2009).

45. E. Salomatina, B. Jiang, J. Novak, and A. N. Yaroslavsky, "Optical properties of normal and cancerous human skin in the visible and nearinfrared spectral range," J. Biomed. Opt. 11(6), 064026 (2006).

46. G. Pellacani and S. Seidenari, "Variations in facial skin thickness and echogenicity with site and age," Acta Derm.-Venereol. 79, 366-369 (1999).

47. S. Diridollou, V. Vabre, M. Berson, L. Vaillant, D. Black, J. M. Lagarde, J. M. Grégoire, Y. Gall, and F. Patat, "Skin ageing: changes of physical properties of human skin in vivo," Intl. J. Cosmetic Sci. 23, 353-362 (2001).

48. C. Eisenbeiss, J. Welzel, W. Eichler, and K. Klotz, "Influence of body water distribution on skin thickness: measurements using highfrequency ultrasound," Brit. J. Dermatol. 144, 947-951 (2001). 